\title{
THE MULTIVERSE: AN EXISTENTIAL CRISIS FOR SCIENCE?
}

For most scientists, it seems, there's always been the hope that the entirety of the scientific endeavour all of the observations, analyses, experiments, and theorizations would eventually lead to an understanding of life and of the Universe, its origins, and, most importantly, its purpose an understanding profound and deep, like a scientific counterpart to the sense of understanding which religions and philosophies have brought to men since times much earlier than the development of science.

Recent discoveries and developments in cosmology and quantum physics have, however, led many of the world's physicists to suggest that the Universe, as we think of it, is not all that exists. Reality, it turns out, might be "far grander" and "far stranger" than previously thought, and, alas, "mostly hidden," perhaps forever, from human observation (Greene 2011: 8) ${ }^{1}$. According to American physicist and writer Alan Lightman's article from Harper's Magazine, titled "The Accidental Universe," also published in the 2012 edition of The Best American Essays collection, "dramatic developments in cosmological findings and thought," mainly those of two particular modern physics theories eternal inflation and string theory as well as the discovery of the accelerated expansion of the cosmos driven by a mysterious "dark energy" and the astonishing apparent "fine-tuning" of its density in space for the emergence of life in the Universe led to the proposition, by many prominent physicists and cosmologists, that the physical reality which we observe and call "The Universe," is actually one constituent part of an "enormous," perhaps "infinite" po-

\footnotetext{
${ }^{1}$ Page numbers provided for Greene are those from the electronic PDF version of the book, as cited. Actual print page numbers are different.
}

pulation of parallel universes "with wildly varying properties," existing "far apart," and/or "simultaneously in time", all collectively known as the "multiverse," and all, including ours, being completely accidentally configured our own existence, in line with Lightman's somewhat poignant title, being nothing more than a "mere [accident] a random throw of the cosmic dice" (Lightman 2012: 207; 209; 213-215). What these proposals suggest, is not simply another addition of another order of magnitude from a galaxies-bearing Universe to a universes-bearing Multiverse. What these proposals suggest, in Lightman's (2012) eyes, is that the traditional role of science to explain why our Universe must have the features it currently has, stemming from the "Platonic" vision of our Universe, as a single, "self-consistent" entity "in which everything could be calculated, predicted, and understood," is "futile, a beautiful philosophical dream that simply isn't true" (p. 213). In a Multiverse involving all physical possibilities, as suggested by some theoretical models, our universe and its features, including its inhabitants-us, are nothing special, and certainly not necessary to exist these are simply one of the endless possibilities, each realized in some universe. These Multiverse proposals have raised many paramount questions and concerns and, not surprisingly, have garnered a strong opposition. Due to the near-infinite, or infinite nature of reality of the proposals being far (or infinitely far) and probably forever beyond the reach of any observation by the Human Race (or any hypothetical observer, for that matter), and thus impossible to directly verify, test, or otherwise directly prove the existence of, many leading scientists in the field, notably South African cosmologist and mathematician George F.R. Ellis, FRS, Hon. 
FRSSAF, have questioned the status of them the proposals as legitimate scientific proposals, much less as scientific theories, and have dismissed them as "scientifically based philosophical speculation," warning that loosening requirements for scientific consideration, in order to include untestable proposals, such as the multiverse, would expose the scientific establishment to a conquest by many other competing but yet unqualified disciplines, like Astrology, that are eager to claim their share of scientific prestige and credibility (Ellis 2011). With many proponents countering the counter-arguments, suggesting indirect and some direct ways in which the proposals could be tested and, thus, be testable, and pointing to the way in which the view of the world in science and the view of science itself and its definition has continuously been reshaped in recent history, the world stage of science is set for a continuous debate, not just about the nature of the proposed theories, but about the nature of science itself, with attitudes ranging "from strong support through open minded agnosticism to strong opposition" (Ellis \& Carr 2008: 2.29). If considered true, however, the Multiverse proposals result in even bigger questions about the place and purpose of Humans in it and whether those are diminished with the further removal from our past anthropocentric worldview, (not to speak of some of the "bizarre" possibilities consequent of physical infinities suggested by the proposals, such as the existence, far in reality or in parallel dimensions, of an infinite number of our clones, ones leading identical lives to ours', others drastically different, and everything in between); questions like whether they make it truly impossible to calculate and fully understand at least our own universe, should it be finite in size; whether they pose a true challenge to the Design Argument for God by apparently explaining "anthropic fine-tunings" of fundamental constants, ${ }^{2}$ whether such challenge might have been

\footnotetext{
${ }^{2}$ According to Lightman, "anthropic, from Greek for 'man,'”
}

the main motivating force behind the proposals, and, even if yes, whether they actually succeed in answering the metaphysical questions they might have been invoked to answer in the first place, regarding the origin of and reason for existence and whether "an accident" might be a mandatory answer to such questions. Although the existence of a Multiverse could be seen as a possible prediction by many modern physics theories, and although it offers solutions for many scientific dilemmas, the near absence of any routes for its direct verification, the multiplicity of possible combinations of its different proposed types, the relative young age and experimental history of the theories that predict its existence, as well as many questions and paradoxes that its existence would raise all of those make the Multiverse still a great proposal for further consideration and indirect testing, but not yet a great candidate as a scientific theory, and close to, but still a bit short of a candidate as a truly scientific proposal. Nevertheless, if considered true, now or in the future, by sudden direct evidence or an alternate way of proving, or by changing the definition of what is meant by 'science, it would still leave unanswered the ultimate questions of origin, existence, and purpose, leaving room for metaphysical and theological explanations, and even, perhaps, hopes of eventual scientific understanding.

"The notionof parallel universes leapt out of the pages of fiction into scientific journals in the 1990s," the "In Brief" section from a Scientific American article states (Ellis 2011). The theory of eternal inflation, one of the theories that predicts a multiverse, came from MIT physicist Alan Guth's inflation theory, a theory of faster-thanlight expansion of the Universe in the very first instant of the infant universe, a theory which he had proposed about 30 years ago, which solved

coined by British physicist Brandon Carter, "is a misnomer: if these fundamental parameters were much different from what they are, it is not only human beings who would not exist. No life of any kind would exist". 
many problems with the normal Big Bang theory, such as the observed homogeneity of the cosmic microwave background radiation (CMBR). Alexander Vilenkin, one of the co-developers of eternal inflation, according to Lightman, explains the theory and how it predicts multiple universes:

Inflation is a period of super-fast, accelerated expansion in early cosmic history. It is so fast that in a fraction of a second a tiny subatomic speck of space is blown to dimensions much greater than the entire currently observable region. At the end of inflation, the energy that drove the expansion ignites a hot fireball of particles and radiation. This is what we call the big bang.

The end of inflation is triggered by quantum, probabilistic processes and does not occur everywhere at once. In our cosmic neighborhood, inflation ended 13.7 billion years ago, but it still continues in remote parts of the universe, and other "normal" regions like ours are constantly being formed. The new regions appear as tiny, microscopic bubbles and immediately start to grow. The bubbles keep growing without bound; in the meantime they are driven apart by the inflationary expansion, making room for more bubbles to form. This never-ending process is called eternal inflation. We live in one of the bubbles and can observe only a small part of it. No matter how fast we travel, we cannot catch up with the expanding boundaries of our bubble, so for all practical purposes we live in a self-contained bubble universe. (Vilenkin\&Tegmark 2011)

These inflationary "bubbles," according to George Ellis, are the "most widely accepted" multiverse proposal, perhaps because, in Brian Greene's words, it provides a "mechanism that can actually generate other universes" (Ellis 2011; Greene 2012). String theory too, however, suggests a Multiverse, as mentioned before. "Originally conceived in the late 1960 s as a theory of the strong nuclear force but soon enlarged far beyond that ambition, string theory postulates that the smallest constituents of matter are not suba- tomic particles like the electron but extremely tiny one-dimensional "strings" of energy... [where] different modes of vibration correspond to different fundamental particles and forces" (Lightman 2012: 216). A musical idea indeed, but string theory did bring its own complication to the table: scientists found out that in order for string theory to work, it would have to have not the three spatial dimensions that we observe, but a whopping seven (p. 216). And, it turned out; there isn't just one way in which those seven dimensions can fold to make a mathematically consistent Universe. There are $10^{500}$ ways ( $p$. 216). That's more than the number of atoms in the Observable Universe (which is only $10^{80}$ ) multiplied by itself six times. Known as the "string landscape," this number practically infinite in Lightman's eyes of possible universes, each with differently vibrating strings, membranes, and multidimensional M-branes (This revised version of String theory is sometimes referred to as Mtheory) differently folded into seven dimensions: each, proponents say, could correspond to an actually existing universe ours being just one created through the eternal inflation process (Greene 2012; Lightman 2012: 216; Papadopoulos 2008)³. Both eternal inflation and string theory are considered to be scientific theories for evidence, one needs to look no further than Lightman's own reference to them as such; and although, according to Lightman (2012), "neither... theory has anywhere near the experimental support of many previous theories in physics," and each, "or both, could turn out to be wrong," which would surely diminish the theoretical support for the multiverse, there still is little debate as to whether any of them are scientific theories (pp. $215,217)$. As for the multiverse proposals themselves the case is different.

\footnotetext{
${ }^{3}$ The Works Cited entry for "Parallel Universes" provides a URL link to the video source originally reviewed, which, as of 09 July 2013, is no longer available in that Web location.
} 
"Remember: Parallel universes are not a theory they are predictions of certain theories," reminds us Max Tegmark, an MIT professor and cosmologist, himself one of the strongest proponents of the multiverse. For him, however, the fact that most of the proposed types of universes might forever remain hidden from our view is not by itself a disqualification of the proposals as science. "To me, the key point is that if theories are scientific, then it's legitimate science to work out and discuss all their consequences even if they involve unobservable entities," he says. The theories he is referring to, off course, are ones like string theory and eternal inflation which predict, as a consequence, the multiverse. He extends his argument by pointing to other unobservable entities that are still considered scientific as part of the package theory with which they came: "For example, because Einstein's theory of general relativity has successfully predicted many things that we can observe, we also take seriously its predictions for things we cannot observe, e.g., what happens inside black holes (Vilenkin\&Tegmark 2011)." His argumentation comes as a direct response to multiverse critic George Ellis, who has argued that "Proponents of the multiverse, as well as greatly enlarging our conception of physical reality, are implicitly redefining what is meant by "science." Essentially his argument stems from the current mode of thinking in science, that if something is unobservable, even in theory, and therefore impossible to prove or disprove by experiment, observation, or otherwise, as we will see is mostly the case with multiverse proposals, then, in general, there is no basis of considering it science.

The basic problem with all multiverse proposals is the presence of a cosmic visual horizon. The horizon is the limit to how far away we can see, because signals traveling toward us at the speed of light (which is finite) have not had time since the beginning of the universe to reach us from farther out. All the parallel universes lie out- side our horizon and remain beyond our capacity to see, now or ever, no matter how technology evolves. In fact, they are too far away to have had any influence on our universe whatsoever. That is why none of the claims made by multiverse enthusiasts can be directly substantiated. (Ellis 2011)

There have been ways suggested, actually, of possibly directly verifying the existence of a multiverse by studying closely the cosmic background radiation, for example. " $[A]$ collision of our expanding bubble with another bubble in the multiverse would produce an imprint in the cosmic background radiation-a round spot of higher or lower radiation intensity. A detection of such a spot with the predicted intensity profile would provide direct evidence for the existence of other bubble universes," Alexander Vilenkin writes (Vilenkin\&Tegmark, 2011). The question then, off course, is whether such a collision has occurred or not, since it is still possible that a bubbles multiverse exists, but that no other parallel universe has ever collided with ours. Ellis still remains skeptical, however, pointing out that "many of the hypothetically possible multiverses would not lead to such evidence. So observers can test only some specific classes of multiverse models in this way" (Ellis 2011). Similarly, he points to the often assumed infinity of space in many proposals of the multiverse, as yet another example of proponents (of the multiverse) crossing the borders of "science:"

What has been forgotten here is that infinity is an unattainable state rather than a large number - its character is totally different from any finite number and it is a mathematical rather than physical entity. According to David Hilbert (1964): "The infinite is nowhere to be found in reality, no matter what experiences, observations, and knowledge are appealed to. "Even if there were an infinite number of galax-ies, and we could see them all (which we could not), we could not count them in a finite time. So there is no way 
the existence of an infinity can ever be proven correct by observation or any other test. The concept of physical infini-ties is not a scientific one if science involves testability by either observation or experiment (Ellis \& Carr: 33) .

The question indeed becomes then "what does science involve, and what should it involve? Does it involve the extrapolation of observable data into the unobservable? Or would such a measure be considered implicit "redefining" of science, as George Ellis suggests? Or, perhaps, is it time to actually review and expand the current borders of the scientific consideration, effectively "redefining," though explicitly, "what is meant by 'science'?" "The proponents [of the multiverse] are telling us we can state in broad terms what happens 1,000 times as far as our cosmic horizon, $10^{100}$ times, $10^{1000000}$ times, an infinity all from data we obtain within the horizon," Ellis (2011) writes. One example of such extrapolation is what Max Tegmark calls "level 1" multiverse, the idea that if our space extends infinitely far beyond the cosmic visual horizon, then, at a certain distance, simply because of mathematical probability, there will be a galaxy with a solar system and a planet identical to ours, with people identical to us living an experience identical to, and/or slightly different than ours (Papadopoulos 2008; Ellis 2011). Even more, there will be an infinite number of such "doppelgangers," as Brian Greene has referred to them, all playing out every possible outcome of our lives an infinite number of times (Greene 2011: 15; Papadopoulos 2008). "But the trouble with this type of extrapolation," to George Ellis, at least, besides his personal opinion that "It is a huge act of hubris to extrapolate from one small domain to infinity when infinity is never attainable,' the trouble "is that no one can prove you wrong" (Ellis \& Carr: 33; Ellis 2011). It is this unfalsifiable nature of the multiverse proposals that alarms many scientists, like Lightman (2012), who concludes that from the multiverse theories it looks like "to explain what we see in a world and in our mental deductions, we must believe in what we cannot prove" (p. 217). Not everybody is distressed, off course. From a proponent's standpoint, Brian Greene a theoretical physicist, string theorist, professor at Columbia University, and author of many books like Max Tegmark, argues that it is the explanatory power of a proposal that matters: "a theory's success can be used as an after-the-fact justification for its basic architecture, even when that architecture remains beyond our ability to access directly (Greene 2011: 195). He shares Bernard Carr's view that historically, "the multi-verse is just one more step in our progress from geocentric to heliocentric to galactocentric to cosmocentric worldview," a completion point of a 500-years-old paradigm shift in our view of our centrality in the Universe known as the "Copernican revolution," named after the Renaissance astronomer who started it by suggesting that the Sun, and not the Earth, was the center of the Universe (Greene 2011: 355-356; Ellis \& Carr: 2.36). Both Greene and Carr point to the changes in the nature of science, more precisely to the expansion of what it encompasses that have coincided with the expanse of what we perceive to be all of reality. For example one seen in Greene's Hidden Reality (2011) during the times of Newton, science dealt with everything tangible and directly observable, from falling apples to the moving planets. During Maxwell's time, however, science started dealing with electromagnetic waves, in a "significant step" towards "abstracttion," while in the 20th century it started describing subatomic phenomena, and ones related to space and time an abstraction of a wholly new order (pp. 194-195). In Carr's example, experimental ability used to be one very important aspect, an importance like that which opponents like George Ellis prescribe to the ability to observe. However, with the advance of Astronomy and Cosmology, especially during the shift to cosmocentric view, it became clear that in order for the 
inclusion of the domains which those two encompass the stars and the galaxies unreachably far in the cosmos under the umbrella of science, one could not require for experimental ability to be a necessary tool available to all proposals that will be considered to be scientific, since scientists could not really perform direct experiments with stars and galaxies. But, luckily for scientists, "Nature effectively performs experiments for us," as Carr puts it, and, with some change in what exactly constituted science, astronomy and cosmology and the respective domains which they observe are today all known to be part of legitimate science (Ellis \& Carr 2008: 2.37). Following these examples and the pattern which they show, we could argue that now we are shifting from a cosmocentric view where the Universe was the center of existence, to a multiverse view where the universe is one of many universes just like our galaxy is one of the many galaxies in existence, and that scientists should make the appropriate changes to the definitions of science, in order to include those other domains in it, if under current thinking they might be considered unscientific. In different words, Brian Greene (2011) asks some important questions in agreement with Carr and in dispute with Ellis that illustrate the same point; questions like, should the direct "scientific" evidence for the proposed other universes continue to be absent, that whether we must perhaps change our view of science itself, and include in the definition of "respectable science" not only ideas and proposals that current technology and theoretical understanding allows us to observe, but also ideas and proposals that might be observable with future technology? Or, even more importantly, he asks, rather poetically, whether perhaps we should "allow science to follow any and all paths it reveals, to travel in directions that radiate from experimentally confirmed concepts but that may lead our theorizing into hidden realms that lie, perhaps permanently, beyond human reach" (p. 360)? Despite his contention about the value of explanatory power that "If the unifying explanation assumes the existence of unobservable entities such as parallel universes, we might well feel compelled to accept those entities," George Ellis's argument against, in this case, is that the number of hypothesized entities ought to be less than the entities or phenomena that they are invoked to explain, while with the multiverse proposals, there is an enormously large, or perhaps infinite number of universes supposed in order to explain our one and only Universe ("It hardly fits 14th-century English philosopher William of Ockham's stricture that 'entities must not be multiplied beyond necessity,"' He writes), and he seriously warns scientists against abandoning of the "core features of science that have led to its phenomenal suc-cess" precisely "the feedback from reality to theory provided by experiment and observational testing" he warns them about abandoning those at their own peril, wary of the possibility, that now, "with the weakened kinds of criteria proposed," pseudo-scientific enterprises like Astrology too could become candidates for scientific recognition. "At the very least," Ellis asserts, "we must be given a clear statement as to what broader definition of the nature of science is being proposed, and in particular what criteria of testing will be taken to be adequate" (Ellis 2011; Ellis \& Carr 2008: 35). There is no doubt that the multiverse proposals are radically differrent than the discoveries of stars and galaxies, or even quantum physicshence all the concerns and the heated debates. Even though there have been some possibilities for some indirect and some direct observations of the proposed entities, as we saw, it is quite possible that no confirmation of any kind, of their existence, will surface for a long time, perhaps ever. What to do? Must we really "believe in what we cannot prove," as Alan Lightman reluctantly suggests? There is certainly some strikingly ironic parallel with the religions themselves the very opposite of what some scientists are trying to disprove (more on that 
later) with the multiverse. "Theologians are accustomed to taking some beliefs on faith," Lightman (2012) writes, but "Scientists are not. All we can do is hope that the same theories that predict the multiverse also produce many other predictions that we can test here in our own universe" (p. 217). But how long, one is compelled to ask, will it take? “...I wonder how long we should wait before a field yields real, experimentally verifiable fruit?" Adam Frank (2010) from National Public Radio's 13.7 Cosmos and Culture Blog asks on the subject. "how much effort do we put into explorations based on the potentially unobservable while shifting away from the tradition of exploring only the actual?... How many universes, ultimately, are enough?” Brian Greene (2011) seems to have an answer: "No one knows whether it will take years, decades, or even longer for observational and theoretical progress to extract detailed predictions from any given multiverse. Should the current situation persist, we'll face a choice," a choice between abandoning the view of the multiverse as science or expanding the definition and scope of science (p. 360). But for now, as reflected in Greene's (2011) following passage, and as felt by Alan Lightman's intuition in his closing passage, all we can do as a generation is wait:

A given generation of scientists can never know whether the long view of history will judge their work as a diversion, as passing fascination, as a stepping-stone, or as having revealed insights that will stand the test of time... determining whether any of these ideas goes beyond mathematical musings of the human mind will require more insight, knowledge, calculation, experiment, and observation than we've so far achieved. A final reckoning on whether parallel universes will be written into the next chapter of physics' story must therefore also await the perspective that only the future can bring. (p. 353)

So the future will hopefully show us whether the multiverse proposals stand as real science, and perhaps are proven, or, at least partially confirmed by indirect experiments. But what about the theoretical framework of the proposals itself? Is it perfect? Is it yet a fully consistent single theory with flawless mathematics and a smooth connection with all other existing theories and knowledge? An interesting documentary on the proposals illustrates and explains intriguingly some of the theories behind them, the arising concepts themselves, and many implications of those concepts explains them it well, with an amateur audience in mind, but nevertheless raises some questions, and, at least seemingly, some paradoxes. According to "Parallel Universes," (an episode from The History Channel's The Universe series, the idea of level three and level four "parallel" universes i.e. universes (infinitely many of which are almost identical to ours) that exist simultaneously in the same space and time as ours that idea is intertwined with the notion that "anything that can happen, has happened or will happen," as expressed by the "Heisenberg uncertainty principle" (Papadopoulos, 2008, mm. 25:38). (The Heisenberg uncertainty principle, a principle in quantum physics, tells us that a given particle in some cases "must be in two places at once," giving rise to arguments that all possible historical outcomes simultaneously exist at once). It is also said that the level 4 Multiverse has universes that have completely different laws of physics and nature (mm. 32:46) (or perhaps they are so different that the terms of physics don't even apply; such universes might not even be comprehensible by our mind). A paradox appears then, at least on a philosophical level, as one is compelled to ask: "What defines 'can happen' (as opposed to 'can't happen') in a Multiverse where each universe is governed by different laws of nature?" (The paradox here is that in a Multiverse with dramatically different universes the realm of possibilities can't really be defined since there is no universal guide of rules that can be used to construct such a definition). Similarly, the argu- 
ments that the conclusion that the Multiverse exists stems from the analysis of the current models of the Big Bang, such as the eternal inflation theory as well as mathematical and logical analysis of the workings of quantum physics (i.e. Heizenger's "uncertainty principle" applied on universal scale to suggest parallel existence) and the related string theory that argument creates another paradox. The paradox, as in the first case, lies in the fact that if different universes exist, where different laws of physics (or completely different laws, or, even no laws at all, since the Multiverse idea is associated with infinity of size and possibilities) then, in theory, there could be Universes, where the analysis of local laws does not yield a Multiverse world. In other worlds, all of the calculations that suggest a Multiverse are calculations of only the laws of our world, and, ironically, the calculations of local laws of some other worlds, the existence of which the concept of Multiverse mandates, those calculations (again, in some yet necessary cases) would not yield a Multiverse, creating a paradox, or, perhaps, a flaw in the suggested concept. Off course, these are only some of the proposed types of multiverses, and the other proposals could still be true, or the explanation could be more complicated than it seems. But the more general point is, that the multiverse is not one single proposal that, at least in theory, is completely crafted, polished, consistent with itself and all the other theories in science. The multiverse proposals are many, with many variations, stemming from many theories, in combination or not. string theory and eternal inflation are not the only sole theories that give rise to the multiverse, but even if we take only them, there are currently many different versions of each theory, that give rise to combined many types of multiverses, all of which could exist, some, or none at all. On one hand, the multiplicity of roads leading to a multiverse could be seen as a point in favor of the proposals, but, there is still a lot of theoretical work that could be done, in order to create much stronger and consistent single theories, or even one and only theory, that would, one hopes, eventually be refuted or accepted.

And What if it is? Let's say tomorrow newspapers headline that scientists in an underground collider laboratory have found evidence for a string-theory based multiverse, or, that astronomers and cosmologists have made observations that without any reasonable doubt prove that we are living in a multiverse. What would that mean? What would the implications be? Would our Universe turn out to be "incalculable by science," as Lightman (2012) suggests (p. 209)? Would that mean that we - all of us - are indeed accidents? Would intelligent design be necessarily ruled out? In other words, what does the multiverse really rule out, without return? One of the other strong motivations for the invocation of a multiverse, besides the modern theories in physics, the math of which leads to such a proposal, has been an amazing set of findings that led to an amazing mystery apparent in the Universe - a "mystery" joyous and totally obvious for the believers in a Creator, but one indeed inexplicable and frustrating for atheistically leaning scientists. The mystery is the apparent "fine-tuning" of the universal constants in physics, fundamental features of our Universe, especially that of the density of the so called "dark energy" - itself an unprecedented recent discovery, an invisible force which propels and accelerates the expansion of the Universe "fine-tunings" towards the emergence and development of life (Lightman 2012: 211-215). Until now, scientists had been unable to explain, strictly in scientific terms, why is it that those fundamental constants and features are exactly in what they discovered to be an extremely narrow range that would allow the formation of galaxies, stars, and thus us. In the example of dark energy, that constant "has been calculated to be ... $10^{-8 "}$ ergs per cubic centimeter" in our Universe, while scientists have established the "theoretically possible amounts" to be from $-10^{115}$ to $+10^{115}$ (214). "If 
the theoretically possible positive values for dark energy were marked out on a ruler stretching from here to the sun, with zero at one end of the ruler and $10^{115}$ ergs per cubic centimeter at the other end, the value of dark energy actually found in our universe $\left(10^{-8}\right.$ ergs per cubic centimeter) would be closer to the zero end than the width of an atom." What could account for such striking fine-tuning? Simple coincidence?A Creator with a plan, perhaps? "Intelligent design is one answer," Lightman (2012) agrees, but it "is an answer to fine-tuning that does not appeal to most scientists" (p. 212). The multiverse-apparently does. It is interesting to consider that for some, the proposal and the advocacy of a multiverse seems to stem exactly from a desire to move away from what otherwise would seem to be potential evidence for the existence for a creator, or at least patterns for meaning and purpose in the Universe. Off course, many might want to dispute the multiverse on that ground as well. But, the real question is, would the multiverse really disprove intelligent design and provide explanations which theology usually does? According to Lightman (2012), because the multiverse would allow all the possible constants and fundamental properties (possible in a sense that they would create some kind of a self-consistent universe), then the fact that our Universe has properties hospitable to life would not require explanation, since, probabilistically, this possibility would have to be realized somewhere, and, the fact that we observe that very possibility is not amazing at all, since, by definition, we would not be able to observe any other ones.

The explanation is similar to the explanation of why we happen to live on a planet that has so many nice things for our comfortable existence: oxygen, water, a temperature between the freezing and boiling points of water, and so on. Is this happy coincidence, just good luck, or an act of Providence, or what? No it is simply that we could not live on planets without such properties.
Many other planets exist that are not so hospitable to life, such as Uranus, where the temperature is -371 degrees Fahrenheit, and Venus, where it rains sulfuric acid... The multiverse offers an explanation to the fine-tuning conundrum that does not require the presence of a designer ( $p$. 212).

There is a little shortcoming, however, at least to those who might hope that the multiverse would close the question of a designer once and for all. The problem is: the multiverse still doesn't rule out the possibility of intelligent design, and, it can be argued, it doesn't even remove the need for a designer. Although its existence might explain what we take to be "finetunings" for the emergence of life as simply one of the realizations of all possible configurations, it would still fall short of explaining the emergence and existence of sentient and intelligent life that is now debating its existence. It would still come short of answering the most fundamental questions that have haunted human beings since the beginning of inquisitive thought, questions for which philosophy and religion have had their own answers, questions like why is there existence at all? Why did the world come into being in the first place? It seems clear after asking these questions that the hypothetical multiverse, though reduces the desperate "need" for a creator, still does not serve as proof against one's existence. It would not logically follow from a multiverse that we are "an accident." Alan Lightman (2012) is well aware of this difference, quoting Steven Weinberg, a Nobel Prize-winning physicist, that "Over many centuries science has weakened the hold of religion, not by disproving the existence of God but by invalidating arguments for God based on what we observe in the natural world (qtd. on p. 212). In this case, even "invalidating" would probably be a too much of a strong word, since all the multiverse would do is move the question of a Universe wholly fine-tuned for life to a multiverse partly, albeit in tiny proportions, 
fine-tuned for life. It would invalidate the argument for God based on perceived fine-tuning for spontaneous creation, by invalidating its premise (since on multiverse scales, the hospitableness to life from just our region would not constitute "fine-tuning") but the complexity of intelligence, sentience, and the existence itself (Universe or Multiverse) would certainly remain unexplained. As Craig Ferguson (2009), a Scottish theologian from University of Edinburgh, has put, "The ensemble [of universes] is not any more self-explanatory than one single universe." (p. 51). As a matter of fact, there is one interesting argument that comes to mind, which some scientists could make in favour of the multiverse potentially explaining the emergence of intelligent life. They could say that since an infinite in size Multiverse would necessarily include all possible physical outcomes at any moment, and since intelligent life is in the realm of possibilities (otherwise we wouldn't be here to think about it), then the Multiverse would effectively explain intelligent life. But here we could hit back at square one by asking the question "why, then does that extremely complex intelligent life and sentience exist in the realm of possibilities?" Again, why does the multiverse exist at all? As Ferguson (2009) notes, again,

Why our multiverse should have a principle of generation that renders probable or inevitable at least one anthropic universe itself requires explanation since it does not appear to be obviously right or at all self-explanatory. As one recent writer puts it, 'even if an inflationary multiverse generator exists, it must involve just the right combination of laws, principles and fields for the production of life-permitting universes'... So while a multiverse theory certainly weakens the anthropic principle, it is not clear whether the hypothesis can render theological explanation redundant. If we have an ensemble of universes that is amenable to scientific description and which is capable of generating one like ours, then we can reasonably ask whether this requires explanation... When all the smoke has cleared, we seem again to be confronted with the twin questions of why there is a universe at all and why it exhibits a rational structure capable of scientific description by its conscious inhabitants. (p. 51)

Of course, we could say all these are rather metaphysical, even philosophical questions. But isn't the purpose, or perhaps the hope of the scientific enterprise to dig deep enough and eventually to be able to answer these questions with scientific certainty? Alan Lightman (2012), for one, thinks that at least "Theoretical physicists... are not satisfied with observing the universe [as opposed to experimental physicists].They want to know why" (p. 208). But perhaps this in itself is part of a bigger, broader issue involving our understanding of the world and our employment, separately or simultaneously, of traditional science, religions and philosophies, and, more recently, the multiverse to that end. When Lightman opens his "The Accidental Universe," he speaks of an important trend that has driven science, and which science has driven, until recently, that seems to have reached its end in relation with the proposals of the multiverse. The trend he speaks of is that of explaining phenomena "once thought to have been fixed at the beginning of time or to be the result of random events thereafter" as "necessary consequences of the fundamental laws of nature - laws discovered by human beings." This is the trend in "The history of science," of "recasting of phenomena that were once thought to be accidents as phenomena that can be understood in terms of fundamental causes and principles. (p. 207)" This trend, according to Lightman, "may be coming to an end," because scientists have realized that, as in the cases of the string theory with its immense "string landscape" and eternal inflation theory with its multiple big bangs, that it is in fact impossible to explain phenomena in our universe as "necessary consequences" of fundamental laws, 
because they are not: the fundamental laws point to almost infinitely multiple possible "consequences," indeed resulting from random processes and all of them, including the ones we observe, being just "mere accidents" (p. 208). He likens us trying to explain our world's properties as necessary "to a school of intelligent fish... hope[ing] to prove that the entire cosmos necessarily has to be filled with water," only to realize that their endeavour has been doomed from the start, their failure stemming from the very erroneous assumption at its base (p. 213). But what if science, as we understand it, was never meant to finish the trend of fundamental explanation. What if it was indeed impossible for science alone to explain the Universe in the most fundamental way? The thought behind this question is that no matter how deep science digs and how deeply fundamental its explanations become, it still seems to fail, always, to answer the most fundamental questions mention above like "why does the world exist, is there any purpose is its existence" or "why is there intelligent and sentient life that is now questioning its own existence?" What if, in order to attempt to answer these questions the scientist has no other choice and simply needs to invoke a metaphysical and/or theological entity like God, or is forced to invoke an unobservable and all-encompassing/all-explaining entity such as the multiverse to avoid marriage with theology, as is described by Bernard Carr's partial contention that "Without a multiverse one may be forced to adopt a non-physical explanation [to fine-tuning] like a fine-tuner, which is why Neil Manson (2003) claims that 'the multiverse is the last resort of the desperate atheist" (Ellis \& Carr, p. 2.37)? And even then, as we saw, the multiverse might not be able to fully address and answer these questions. George Ellis (2011) reflects on this issue as well, finding both that the multiverse ultimately fails to address these fundamental questions, and, for that matter, that they are indeed by definition beyond the scope of science:
Physicists' hope has always been that the laws of nature are inevitable that things are the way they are because there is no other way they might have been but we have been unable to show this is true. Other options exist, too. The universe might be pure happenstance it just turned out that way. Or things might in some sense be meant to be the way they are - purpose or intent somehow underlies existence. Science cannot determine which is the case, because these are metaphysical issues.

Scientists proposed the multiverse as a way of resolving deep issues about the nature of existence, but the proposal leaves the ultimate issues unresolved. All the same issues that arise in relation to the universe arise again in relation to the multiverse. If the multiverse exists, did it come into existence through necessity, chance or purpose? That is a metaphysical question that no physical theory can answer for either the universe or the multiverse.

All in all, it seems evident that the multiverse proposals, whether motivated by an anti-theological disposition or whether simply believed in on the basis of the theories which put it forward it seems evident that even if accepted as science, these proposals will not by themselves rule out the possibility of intelligent design, nor will they by themselves be able to cover all those fundamental questions which science has been trying to answer and which religions and philosophies have covered on their own. What is evident is that the multiverse, if taken to be real, will almost certainly expand not just our perception of physical reality, but also the scope of science, as discussed, and, interestingly, the perspective of philosophies and religions. God, as seen by the believers in a creator, has not only our Universe to take care of, but an infinity of others as well. Speak of multitasking. Jokes aside, Nathan Schneider, a writer in the online science magazine SEED, has written in an article on the subject that "Among the scientists and theologians fo- 
cused on the theological consequences of multiverse theory, many of them believe that it actually expands the job description for god" (Schneider 2009). This article not only suggests, surprisingly, that there are many theologians who embrace the multiverse, but that they, actually along with many scientists, are seriously focusing on what the theological consequences of such a major scientific proposal would be. From informative presentations like one titled "Does God Love the Multiverse," to booklets like the one reading "Who's Afraid of the Multiverse" there is a lot showing that a certain part of the scientifically literate theological community is not only not giving up on their beliefs, and/or not blindly refusing the new proposals, but is also re-gearing themselves to this new worldview and imagining its mechanism in a whole new way. In one extreme case Robin Collins, a professor of philosophy at Pennsylvania's Messiah College, “imagines farflung civilizations in the multiverse all in need of salvation and a multiplicity of Christs who would change forms to meet each universe's redemptive needs. 'If you had Klingons somewhere - of course a very fallen race, as we know from Star Trek,' Collins adds, 'God takes up their nature, and there's a Klingon version of the Son'" (qtd. in Schneider, 2009). Whether we will ever get to hear stories of the Klingon version of Jesus (or whatever his name would be) or the Na'vi embodiment of the Virgin Mary will off course depend on our future prospect of crossing inter-universal boundaries, it does definitely help to use one's imagination when trying to comprehend this seemingly incomprehensible concept. After all, some of the proposals put absolutely no limits on the extent of possibilities.

"Possibilities," in fact, might be a good word to describe what these multiverse proposals really bring to the table. Still scientifically unconfirmed, and still, to use a computer term, in "Beta" stage of theoretical understanding (although in computers, "Beta" is used to describe a stage of testing - a status we are unfortunately unable to give to the proposals at least now), they are indeed a set of very imaginative and unprecedented in scope possibilities of what our reality might really be part of. They are not just imaginative possibilities - thought up for the sake of mental entertainment. They are possibilities that arise from analysis of our current understanding of the world, its composition and its mechanism of origin. Unfortunately they are not possibilities that we could confirm or reject with scientific observations and experimentations, and there are good chances that this will remain so for a very long time - indefinitely long. But it does not have to be so forever. There have been some experiments and observations suggested, that could provide much stronger basis for believing in these possibilities. One is the mentioned analysis of Cosmic Microwave Background Radiation (CMBR) that could one day show signature of a collision with another "bubble universe" from the suggested eternal inflation theory. Another one, mentioned in "Parallel Universes," is the ongoing effort at the Large Hadron Collider (LHC) - the world's largest, according to Wikipedia, and highest-energy particle accelerator located 574 feet under the Franco-Swiss border the effort to find indirect evidence for other dimensions suggested by string theory by colliding elementary particles at near-light speeds and later reconstructing the collision with the help of high-tech computers, with close attention to the movement of all of the energy and momentum resulting from it; If the scientists there ever find missing a piece of energy or momentum from the reconstructed result, with no way to account for its loss, then, it has been suggested, they could conclude that extra dimensions exist and that they are exactly where that piece of the missing energy went; the "graviton," a theorized gravity-carrying particle, is said to "know" about the proposed extra dimensions, and, according to scientists, will most definitely "move off" into those extra dimensions, if 
it is ever created in the process of collisions, accounting for the dent in the reconstruction of energy and momentum. Additionally, one can find examples of scientists devising experiments and tests that were once thought impossible, probing in the process entities once thought unobservable or nonexistent. In one such example, according to an article (2011) from "Too Hard For Science?" series from the Scientific American blog "Assignment: Impossible," scientists have proposed examining very weak signals like "neutrinos and gravitational waves," with instruments that might be available in a few decades, to find "traces" of not only the immediate postBig Bang era currently unobservable, but also, as crazy and nonsensical as it sounds, an era before the Big Bang. This could mean, that although the proposed multiverse is currently considered unobservable and assuming the above-mentioned experiments fail to find any evidence for it unscientific according to traditional definitions of science, there could still be possibility for experiments devised in the future, that would be able to more directly probe and confirm/refute the existence of the proposed alternative universes. In the meanwhile, though, while the experimental scientists are busy colliding protons and antiprotons at the LHC, closely scanning the latest images of the CMBR, or thinking up the next unthinkable experiments and instruments to probe the unimaginable and incomprehensible, there is plenty of time for the theoretical physicists to polish and perfect their proposals and their theories, perhaps giving us hope that one day they will come up with the Theory of the Multiverse, beautiful and flawless, but, more importantly one

\section{REFERENCES}

1. Choi, C.Q. (2011, July 19). Too hard for science? Detecting signals before the Big Bang. Assignment: Impossible [Blog]. Scientific American. Retrieved from. and only, one which would sit on its papers then, waiting for the right experiments and/or evidence to come up, finally, glorifying its existence or throwing it into the trashcan of mistaken science, clearing the table up for the next best candidate for the explanation of the world. As for us, we should fully embrace the offered possibilities as something that might be considered true one day, light-heartedly entertaining all the bizarre and science-fiction-ish possibilities they could entail (shout out to my doppelganger who proudly finished his paper two months ago), while appreciating even more the mysterious but in either case grand Universe we are living in, at home or in the labs, making sure we put even more effort into uncovering its own secrets, looking to see if somewhere we might have neighbours that could teach us a thing or two about our world before enlightening us on the nature and the expanse of reality. Brian Greene put it well:

Some people recoil at the notion of parallel worlds; as they see it, if we are part of multiverse, our place and importance in the cosmos are marginalized. My take is different. I don't find merit in measuring significance by our relative abundance. Rather, what's gratifying about being human, what's exciting about being a part of the scientific enterprise, is our ability to use analytical thought to bridge vast distances, journeying to outer and inner space and... perhaps even beyond our universe. For me, it is the depth of our understanding, acquired from our lonely vantage point in the inky black stillness of a cold and forbidding cosmos that reverberates across the expanse of reality and marks our arrival (Greene 2011: 14).

http://blogs.scientificamerican.com/assign ment-impossible/2011/07/19/too-hard-forscience-detecting-signals-from-before-thebig-bang/. 
2. Ellis, G.R. (2011). Does the Multiverse Really Exist? (Cover Story). Scientific American. 305.2 38-43.

3. Ellis, G.R., Carr, B.J. (2008). Universe Or Multiverse?. Astronomy \& Geophysics 49(2), 2.29-2.33. doi: 10.1111/j. 14684004.2008.49229.x.

4. Ferguson, D. (2009). Faith and its critics: A conversation. New York, NY: Oxford University Press. PDF.

5. Frank, A. (2010, March 16). One Universe Too Many? 13.7: Cosmos and culture: Commentary on science and society. National Public Radio. Retrieved from http://www.npr.org/blogs/13.7/2010/03/o ne_universe_too_many_string_t.html

6. Greene, B.R. (2011). The hidden reality: Parallel universes and the deep laws of the cosmos. New York, NY: Random House Digital, Inc. PDF.

7. Greene, B.R. (2012, February). Brian Greene: Is our universe the only universe? [Video file.] Retrieved from: http://www.ted.com/talks/brian_greene_w hy_is_our_universe_fine_tuned_for_life

8. Lightman, A.P. (2012). The accidental universe. In D. Brooks (Ed.), The best american essays, 2012 (pp. 207-217). Boston, MA: Mariner.

9. Papadopoulos, A. (Writer \& Director). (2008). Parallel Universes [Television series episode]. In S. Berkemeier (Producer), The Universe. NewYork, NY:History Channel. Retrieved from:

http://www.history.com/shows/theuniverse/videos/the-universe-paralleluniverses.

10. Schneider, N. (2009, March 30). The multiverse problem. SEED. Retrieved from http://seedmagazine.com/content/article/t he_multiverse_problem/

11. Vilenkin, A. \&Tegmark, M. E. (2011, July 11). The case for parallel universes. Scientific American. Retrieved from: http://www.scientificamerican.com/article /multiverse-the-case-for-parallel-universe/.

BZNUNI VAHAN

\section{THE MULTIVERSE: AN EXISTENTIAL CRISIS FOR SCIENCE?}

\section{SUMMARY}

This paper is a response to an article by American physicist andprofessor at the Massachusetts Institute of Technology (MIT) Alan Lightman, titled "The Accidental Universe." Originally published in Harper's Magazine, "The Accidental Universe," is about the idea of a "multiverse" - a model of reality, in which our known "universe" is only a tiny, and insignificant member of an ensemble of many, perhaps an infinite number of universes. The explored topics in the paper include: the scientific theories that predict such a multiverse; the inherent obstacles to observing that multiverse in order to establish it as a scientific theory; the opposition from some scientists to the recognition of the multiverse as a valid theory; the implications of its possible acceptance as such for the scientific community, and the various philosophical questions, as well as fantastical possibilities that its existence could entail. Different possibilities and theoretical models are explored, and, finally, a perspective is offered for the near future in approaching the multiverse both from a scientific standpoint, and as humans in general.

Key concepts: multiverse, Big Bang theory, eternal inflation, string theory (M-theory), 
anthropic "fine-tuning" of the universe, dark energy.

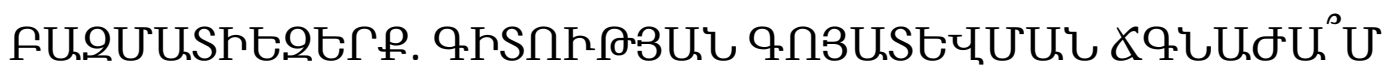

\section{UUФกФПగU}

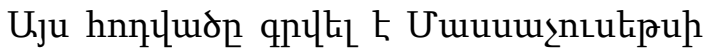

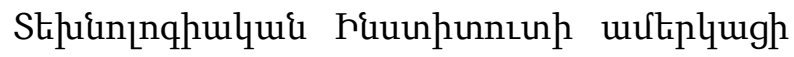
\$hqhlynu, upn\$tiunp U.ui Luppoftip «Tu-

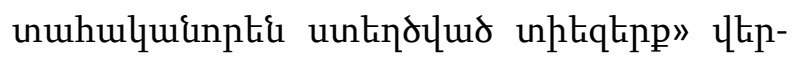

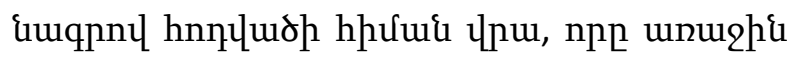

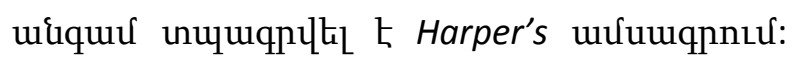
"Tuunuhulquinptí uutindцuð unptiqkpp»"

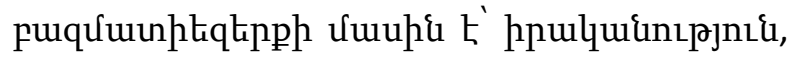
npuntin utiq pninphu hujunup «nptiqtppn»

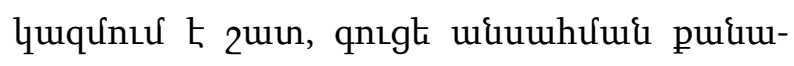

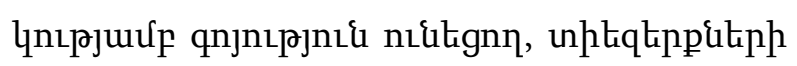

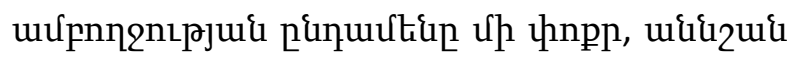

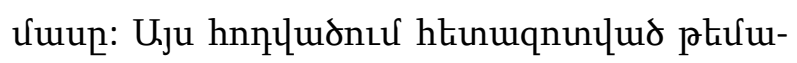

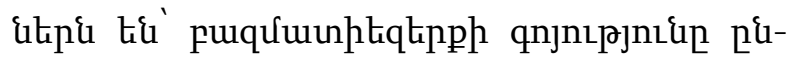

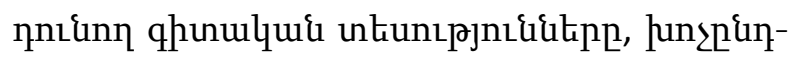
nuiltpn, npnip $\mathrm{h}$ hujun til quihu puqưuinht-

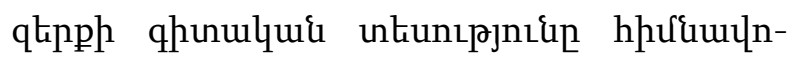

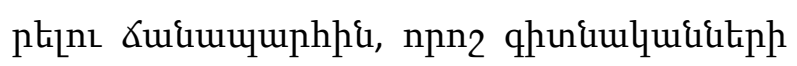

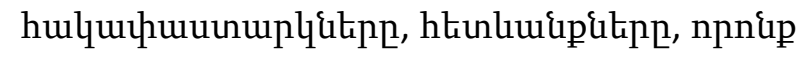
qunnn tis $\mathrm{h}$ hujun quil qhunulquil uhqurluj-

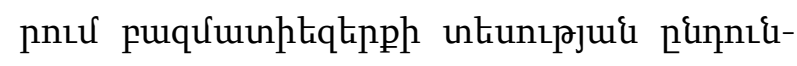

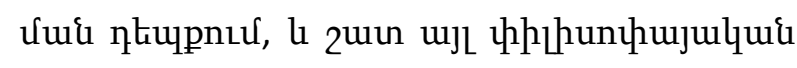

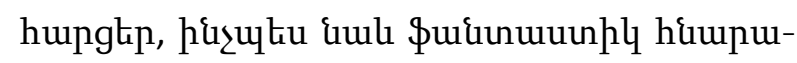

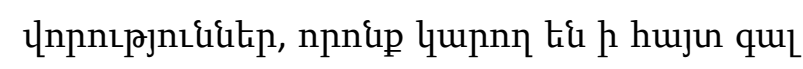

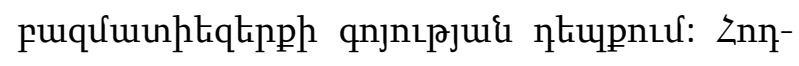

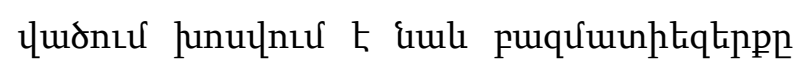
hisuliu qhunulqui, ujaultu hl unopju hơu-

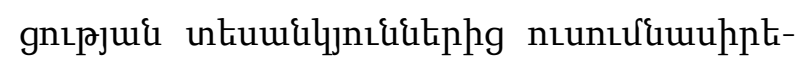
[nı htinuilyuph ưuups:

zuiqqnıgujhe huuqugnıpjnı\&itip. Fuquwunhtiqtipp, fhq ftiqh untiunıpjnıl, huulkndulqui ha\$juighw, juph untunıpjnı (Mtheory), untiqtiph uupnluujhq "fine-tuning», unıp tutinqhuikin: 